\title{
Word Sequences and Intersection Numbers
}

\author{
Jane Gilman * and Linda Keen ${ }^{\dagger}$ \\ Department of Mathematics, Smith Hall, Rutgers University, Newark, NJ 07102 \\ Mathematics Department, CUNY Lehman College, Bronx, NY 10468, USA
}

\begin{abstract}
There are certain sequences of words in the generators of a twogenerator subgroup of $S L(2, \mathbb{C})$ that frequently arise in the Teichmüller theory of hyperbolic three-manifolds and Kleinian groups. In this paper we establish the connection between two such families, the family of Farey words that have been used by Keen-Series to understand the boundaries of the Quasifuchsian space of surfaces of type $(1,1)$ and the Schottky space of surfaces of type $(0,4)$ and the sequences of words that arise in discreteness algorithms for two-generator subgroups of $S L(2, \mathbb{C})$ studied by Rosenberger-Purzitsky and Gilman-Maskit and Jiang.
\end{abstract}

\section{Introduction}

There are certain sequences of words in the generators of a two-generator group that frequently arise in the Teichmüller theory of hyperbolic threemanifolds and Kleinian groups. In this paper we establish the connection between two such families, the family of Farey words that have been used by Keen-Series to understand the boundary of the Teichmüller spaces of surfaces of type $(1,1)$ and $(0,4)$ and the sequences of words that arise in two-generator discreteness algorithms studied by Rosenberger-Purzitsky and Gilman-Maskit and Jiang. In the case that the group under consideration is discrete, this connection allows us to interpret the exponents that arise in the

*Supported in part by a grant from the Rutgers Research Council

${ }^{\dagger}$ Supported in part by a PSC-CUNY grant 
algorithmic words and in their Farey expansions as self-intersection numbers of certain curves on the quotient surface.

Our first main result is theorems 5.1 and 5.2, which connect several sequences of integers that arise in words considered by the algorithm, pairs of primitive associates in a two-generator free group, Farey expansions, and continued fraction expansions. Theorem 6.1 interprets these sequences as unwinding and winding numbers for one curve on the surface about another curve on the surface. These sequences give what we call the complexity of a curve on a surface; we will see that it is related to the complexity of the discreteness algorithm. All the different approaches and their related sequences are summarized in theorem 7.1.

A geometrically motivated algorithm for deciding whether or not a twogenerator real matrix group is discrete was described by Gilman-Maskit [4] The geometric form of the algorithm built upon algebraic forms developed by Purzitsky and Rosenberger [15] and [16] and an incomplete approach due to Matelski [14].

Initially, the Gilman-Maskit geometric algorithm distinguishes between the case that the given generators are hyperbolic with intersecting axes and all other cases, called the intertwining cases [2]. In this paper, we consider the intertwining cases with particular emphasis on the the case when the algorithm decides that the group is free. In this case the quotient is topologically a three punctured sphere; conformally the punctures are usually holes. The axes of hyperbolic generators project onto closed geodesics on the quotient manifold; parabolic elements appear if there are punctures. Discreteness questions in the intersecting axes cases are treated in $[2,8,9,16]$.

The algorithm steps include two types of steps that change the generating pair, depending upon whether the lengths of the words as words in the initial generators grow linearly or exponentially, called non-Fibonacci and Fibonacci steps $([3,18])$. The complexity of the algorithm can be bounded in terms of the numbers of consecutive Fibonacci and non-Fibonacci steps and the traces of the original generators and their product.

Specifically the algorithm determines a sequence of integers defined by the numbers of consecutive steps between Fibonacci steps called the F-sequence. From the F-sequence we obtain the complexity of the projections of the original generating axes. We will show how the F-sequence describes precisely how each such geodesic winds around simpler geodesics and intersects itself along geodesics orthogonal to the simple curves.

Our techniques involve relating the generator sets of a free group on 
two generators produced by the algorithm, the "Farey words" obtained from cutting sequences on a once punctured torus, $[10,11,17]$, the relation of these words to continued fractions and the non-simplicity of the projections of the axes of these words on the underlying surface as determined by selfintersection and the way the curve winds about the holes in the surface.

The organization of the paper is as follows. In section 2 we define basic concepts and notation. In this section we also review some facts about the free group $F_{2}$ and give a canonical form for its primitive elements. In this section we also define the Farey words and their relation to continued fraction expansions and establish our first connection, that between Farey words and primitive elements of $F_{2}$. This introductory section also reviews the algorithm for a pair of hyperbolic generators with disjoint axes. The review emphasizes portions of the algorithm that we subsequently use. In particular, using the F-sequence, we develop a recursive formula for the generating pairs the algorithm considers, corollary 2.8. Finally, in proposition 2.9 we show how the F-sequence and the sequence of primitive exponents in the primitive words are related.

With the background laid out, we are able to discuss the relation between the F-sequences and intersection properties of geodesics. In section 3 we define essential self-intersections and in section 4 we define winding and unwinding for geodesics encountered by the algorithm. In section 5 we establish two main connection theorems: theorem 5.1 and theorem 5.2. In section 6 we derive a recursive formula, theorem 6.1, for counting the number of essential self-intersections of a curve as it winds. Finally in section 7 we pull together all of the connections into one theorem, the main theorem, theorem 7.1. The last section 8 extends the results to groups with parabolic elements and/or generators.

\section{Background and notation}

\subsection{Möbius Groups}

Let $\mathbb{M}$ denote the group of all Möbius transformations of the extended complex plane $\overline{\mathbb{C}}=\mathbb{C} \cup\{\infty\}$. We associate with a Möbius transformation

$$
f=\frac{a z+b}{c z+d} \in \mathbb{M}, a d-b c=1,
$$


one of the two matrices that induce the action of $f$ on the extended plane

$$
A=\left(\begin{array}{ll}
a & b \\
c & d
\end{array}\right) \in \mathrm{SL}(2, \mathbb{C})
$$

and set $\operatorname{tr}(f)=\operatorname{tr}(A)$ where $\operatorname{tr}(A)$ denotes the trace of $A$. For a two generator group, once a matrix corresponding to each generator is chosen, the matrices associated to all other elements of the group are determined.

If the traces of all the elements of a subgroup $G$ of $\mathbb{M}$ are real, then the group leaves some disk invariant. A subgroup $G$ of $\mathbb{M}$ is discrete if no sequence of distinct elements of $G$ converges to the identity. If the traces are all real and the group is discrete, it is called Fuchsian. Every Fuchsian group is conjugate to a group that leaves invariant the unit disk or one that leaves the upper half plane invariant.

In this paper we consider two generator groups that are potential Fuchsian groups. Thus there is no loss of generality in assuming that both generators fix the unit disk (or equivalently the upper half plane).

Below, we will use only the notation $A, B, \ldots$ for elements of $G$.

\subsection{Hyperbolic geometry}

We will use both the unit disk model and the upper half plane model for the hyperbolic plane $\mathbb{H}^{2}$ and denote either by $U$. The Möbius transformations $A$ act as hyperbolic isometries. They are classified as elliptic, parabolic or hyperbolic as the trace satisfies $\operatorname{tr}^{2} A$ is less than, equal to or greater than 4 .

A hyperbolic transformation $A$ has two distinct fixed points on the boundary of $U$ and it leaves its axis, that is, the geodesic joining these points, invariant. It moves points on the geodesic a certain fixed distance in the non-Euclidean metric. This distance will be denoted by $d_{A}$ and is called the translation length of $A$. The axis of $A$ will be denoted by $A x_{A}$.

If $M$ and $N$ are two distinct hyperbolic lines, then either $M$ and $N$ are disjoint or they intersect in a single point interior to or on the boundary of $U$.

If $M$ and $N$ are non-intersecting geodesics with distinct end points there is a unique common orthogonal. If we let $R_{M}, R_{N}$ be reflections (inversions) in these geodesics, then $A=R_{M} R_{L_{N}}$ is a hyperbolic transformation, $A x_{A}$ is the common orthogonal to $M$ and $N$ and $\frac{d_{A}}{2}$ is the length of the segment of $A x_{A}$ between $M$ and $N$. 
Conversely if $A$ is any hyperbolic transformation and $p$ is any point on $A x_{A}$ with $L$ a geodesic through $p$ perpendicular to $A x_{A}$, there is a unique hyperbolic line $L_{A}$, also perpendicular to $A x_{A}$, such that $A$ factors as $A=$ $R_{L} R_{L_{A}}$. We use the convention, apply the rightmost reflection first. The distance between $L$ and $L_{A}$ along the axis of $A$ is $d_{A} / 2$. Transformations that are parabolic and elliptic also factor as the product of reflections in two lines that meet either at a point on the boundary of $U$ or interior to $U$.

Any three pairwise disjoint hyperbolic lines either bound a region in $U$ or one line separates the other two.

\subsection{The free group on two generators}

Let $W=W(A, B)$ denote a word in the generators $A$ and $B$ of the abstract group $F_{2}=<A, B>$ so that $W(A, B)=A^{u_{1}} B^{v_{1}} A^{u_{2}} \ldots B^{v_{r}}$ for some integers $u_{1}, \ldots, u_{r} ; v_{1}, \ldots, v_{r}$.

Primitive words in $F_{2}$ are words that can be extended to a minimal set of generators for the group. These words have a canonical form derived from an analysis of $\operatorname{Aut}\left(F_{2}\right)$ (see problem \# 3 p. 169 of [12])

Theorem 2.1. Every primitive word in $F_{2}$ has the form, up to interchange of $A$ and $B$,

$$
W(A, B)=A^{u_{1}} B^{v_{1}} A^{u_{2}} \ldots B^{v_{t}}
$$

for some $t$, where the $u_{i}$ are all equal to each other and are all either 1 or -1 , the $v_{i}$ all have the opposite sign to the $v_{i}$, and $\left|v_{i}-v_{i+1}\right| \leq 1$.

A pair of primitive words that generate $F_{2}$ are called an associate primitive pair. The pairs that arise in the algorithm are examples of associate primitive pairs. We will see that the form we obtain for the algorithm words in proposition 2.9 agrees with the form in this theorem. In particular, the values of $t$ and the exponents $v_{i}$ can be computed directly from the algorithm steps.

\subsection{Farey diagrams and continued fractions}

In this section we find another form for associate primitive pairs using the theory of cutting sequences applied to punctured tori developed by Series (see [11]).

We begin by defining concepts defined originally by Farey (see [17] for a modern account). 
- We define Farey Addition of rationals as

$$
\frac{p}{q}+\mathcal{F} \frac{r}{s}=\frac{p+r}{q+s}
$$

and we call two rationals Farey neighbors if $|p s-q r|=1$.

Note that if, for a pair of Farey neighbors, $\frac{p}{q}<\frac{r}{s}$, then $\frac{p}{q}<\frac{p}{q}+\mathcal{F} \frac{r}{s}<\frac{r}{s}$ and $\frac{p}{q}, \frac{p}{q}+\mathcal{F} \frac{r}{s}$ and $\frac{p}{q}+\mathcal{F} \frac{r}{s}, \frac{r}{s}$ are both pairs of Farey neighbors.

- We define the Farey level of the rationals $\frac{n}{1}$ for all $n$ to be 0 .

If $\frac{m}{n}=\frac{p}{q}+\mathcal{F} \frac{r}{s}$ for a pair of Farey neighbors, we define the Farey level of $\frac{m}{n}$ to be 1 plus the bigger of the Farey levels of $p / q$ and $r / s$.

- Define the Farey words of $F_{2}$ inductively as follows:

$$
W_{1 / 0}(A, B)=A^{-1} \text { and } W_{0 / 1}(A, B)=B
$$

and if $\frac{m}{n}=\frac{p}{q}+\mathcal{F} \frac{r}{s}$ for Farey neighbors, $\frac{p}{q}, \frac{r}{s}$ with $p s-q r=1$ define

$$
W_{m / n}=W_{r / s} W_{p / q}
$$

- Note that the word length of $W_{p / q}$ is $p+q$. We call $p / q$ the slope of $W_{p / q}$.

To connect the Farey words to continued fraction expansions draw the Farey Diagram in the upper half plane $U$ by joining all pairs of Farey neighbors by hyperbolic geodesics. These geodesics are all disjoint and meet only at common end points. They form an infinite Tesselation of $U$ by geodesic triangles with vertices on $\mathbb{R}$.

Given a rational $p / q$, its continued fraction is

$$
\frac{p}{q}=n_{0}+\frac{1}{n_{1}+\frac{1}{n_{2}+\frac{1}{n_{3}+\cdots+\frac{1}{n_{k}}}}}=\left[n_{0}, \ldots, n_{k}\right]
$$

where $n_{0}$, the integer part, is arbitrary and the remaining $n_{i}$ are non-negative. The $n_{k}$ term is ambiguous: if $n_{k}=1$, we may delete it and increment $n_{k-1}$ by 1 .

We can read off the continued fraction expansion from the Farey diagram as follows: Let $C$ be the geodesic from $\frac{p}{q}$ that is orthogonal to the line $\Im$ 
joining $\frac{0}{1}$ and $\frac{1}{0}=\infty$. As $C$ goes from $\Im$ to $\frac{p}{q}$ it passes through a sequence of triangles $T_{i}$. As it passes through a triangle if there is a single vertex on the left and two vertices on the right write $L$ otherwise write $R$. Since we end in a vertex, there is again an ambiguity at the last step. Write the sequence obtained from $C$ in this way by $L^{m_{0}} R^{m_{1}} \ldots L^{m_{j}}$. Then one can prove $([17])$

Theorem 2.2. If $L^{m_{0}} R^{m_{1}} \ldots L^{m_{j}}$ is the sequence obtained as above from $\underline{p}=\left[n_{1}, \ldots, n_{k}\right]$ then $j=k$ and $m_{i}=n_{i}, i=0, \ldots r$. Moreover $\sum_{0}^{k} m_{i}$ is the Farey level of $\frac{p}{q}$.

We can enumerate these special words by Farey level using Farey neighbors.

Theorem 2.3. [11] Let $p$ and $q$ be non-negative integers with $(p, q)=1$. There is, up to cyclic permutation and inversion, a unique shortest word such that if $0 \leq p / q \leq 1$ it has the form

$$
W(A, B)=A^{-1} B^{n_{1}} \ldots A^{-1} B^{n_{p}}
$$

where $\sum_{1}^{p} n_{i}=q$ whereas if $p / q>1$ it has the form

$$
W(A, B)=B A^{-n_{1}} \ldots B A^{-n_{q}}
$$

where $\sum_{1}^{q} n_{i}=p$. If $p / q$ is a negative rational we have $W_{p / q}(A, B)=$ $W_{-p / q}\left(B^{-1}, A\right)$

Since the Farey words have the canonical form for primitive words, we can combine this theorem with theorem 2.1 to obtain our first theorem connecting the word forms for primitive pairs of generators and Farey neighbors.

Theorem 2.4. If $\frac{p}{q}, \frac{r}{s}$ are Farey neighbors then the words $W_{p / q}, W_{r / s}$ are an associate primitive pair for $F_{2}=<A, B>$. Moreover if $L, M$ is an associate primitive pair for $F_{2}=<A, B>$, then there is an element $C \in F_{2}$ and a pair $\frac{p}{q}, \frac{r}{s}$ of Farey neighbors such that $C L C^{-1}=W_{p / q}^{ \pm 1}$ and $C M C^{-1}=W_{r / s}^{ \pm 1}$.

\subsection{Normalized stopping points}

For $A, B \in \mathbb{M}$ that fix $U$, let $G=<A, B>$ be the group generated by $A$ and $B$. Assume $G$ is non-elementary. If $L$ is the common perpendicular to $A x_{A}$ and $A x_{B}$, we call the three lines, $L, L_{A}, L_{B}$ the reflection axes. A pair of hyperbolics $A, B$ is normalized if $\operatorname{tr} A \geq \operatorname{tr} B>2$; and if, in the unit disk 
model, $L$ is the real axis, $A x_{A}$ lies to the left of $A x_{B}$ in the unit disk, the attracting fixed points of $A$ and $B$ lie above $L$ and $L_{A}$ and $L_{B}$ lie below $L$, see figure 1 . Since $G$ is a subgroup of index at most two in $\tilde{G}$ the group generated by the reflection in the three reflection axes, $\left\{L, L_{A}, L_{B}\right\}$, the two groups are simultaneously discrete or not discrete. When $G$ is discrete, we let $S=U / G$ and $\pi$ be the projection, $\pi: U \rightarrow S$.

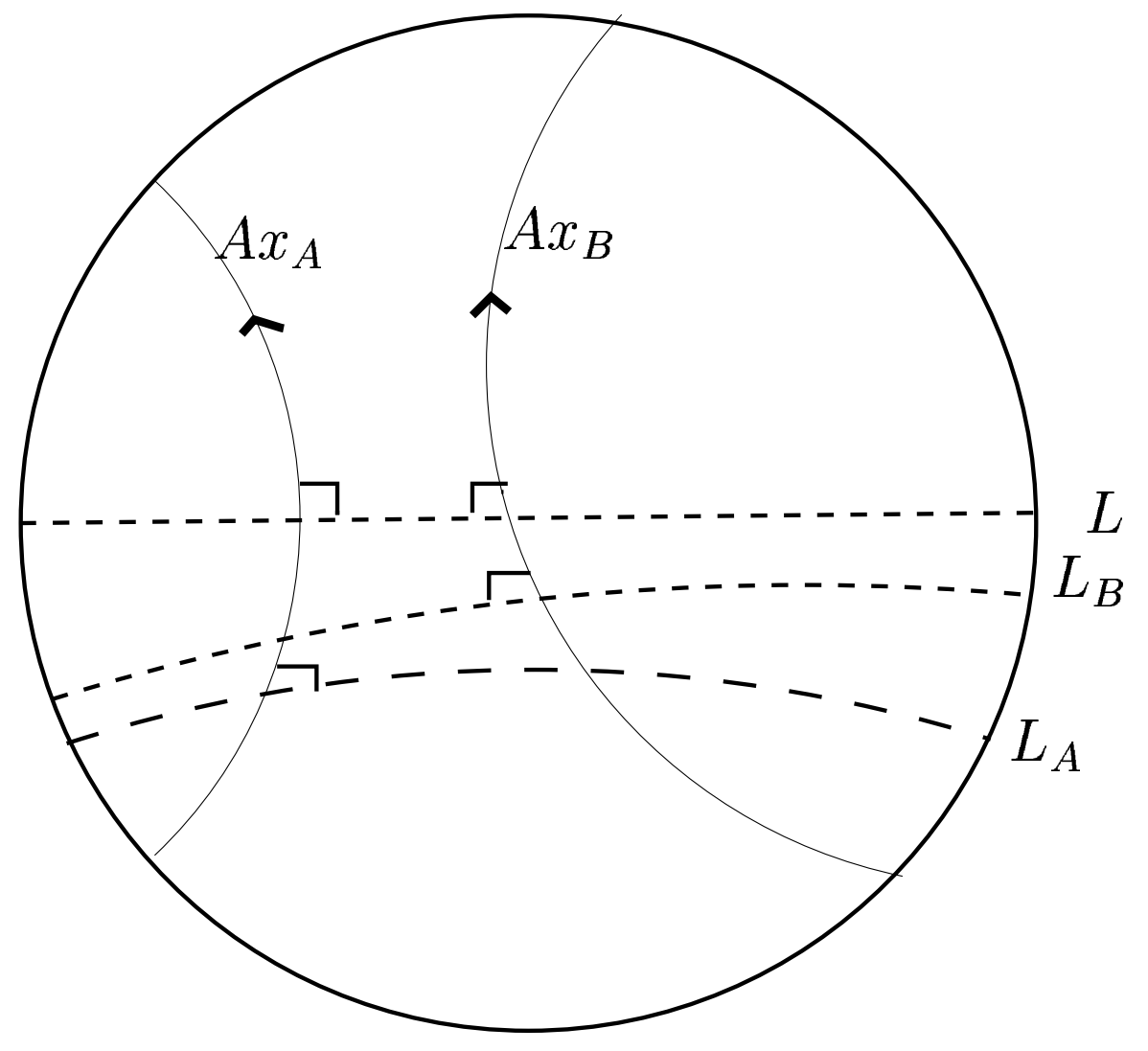

Figure 1: Normalized generators $A, B$ with oriented axes and reflection lines. The axes are solid and the reflection lines are dotted.

Theorem 2.5. [4] If $A$ and $B$ are normalized then $A x_{A B}$ separates $A x_{A}$ and $A x_{B}$. The group $G$ is discrete if $A x_{A}, A x_{B}$ and $A x_{A^{-1} B}$ bound (a region). Further the three axes bound a region if and only if the corresponding reflection axes bound a region. 
Corollary 2.6. ([4]) Stopping point. When the algorithm stops at the pair of hyperbolics $A_{n}, B_{n}$ and says that $G$ is discrete, the corresponding three reflection axes bound a region and the axes of $A_{n}, B_{n}$, and $A_{n}^{-1} B_{n}$ project to a triple of pairwise disjoint simple closed curve on the quotient surface $S=U / G$. These are the shortest curves that the algorithm encounters.

Since the algorithm is trace minimizing, the three simple curves are shorter than any other curves that the algorithm encounters.

\subsection{The Algorithm}

In this paper, we begin by considering groups generated by pairs of hyperbolics and we assume that when the group is discrete it contains no elliptic elements and no parabolics. We treat the case where the two axes are disjoint. In section 8 we extend the treatment to groups containing parabolics, including the possibility that either or both of the generators are parabolic. All groups are assumed non-elementary. We summarize the geometric discreteness algorithm for pairs of hyperbolics with disjoint axes:

The input to the algorithm is a pair of generators $A, B \in \mathbb{M}$ for a nonelementary group $G$. These are normalized and labelled as in section 2.5 so that $\operatorname{tr} A \geq \operatorname{tr} B>2$. The algorithm tests these generators and either (1) it stops and prints out that the group $G$ is discrete or that the group $G$ is not discrete or (2) it replaces the generating pair by a next pair and goes back to the test. The next pair is a specific normalized Nielsen equivalent generating pair. The algorithm guarantees that after a finite number of replacements, a pair of generators is found which stops the algorithm.

Thus the algorithm determines a sequence of normalized generating pairs,

$$
\left(A_{0}, B_{0}\right) \rightarrow\left(A_{1}, B_{1}\right) \rightarrow\left(A_{2}, B_{2}\right) \rightarrow \cdots \rightarrow\left(A_{n}, B_{n}\right) .
$$

The test step considers the ordered pair $A, B$ and looks at $A^{-1} B$. If $A^{-1} B$ is not hyperbolic, the algorithm branches to cases we don't consider here. If it is, and if $\operatorname{tr} A^{-1} B<-2$ it stops and the group is discrete. This is equivalent to the reflection lines $\left(L, L_{A}, L_{B}\right)$ bounding a region. If $2<\operatorname{tr} A^{-1} B<C_{0}$ where $C_{0}$ is a constant derived from Jorgensen's inequality, it stops because the group is not discrete.

The algorithm replaces a pair of generators $(A, B)$ by a next pair of generators that is one of $\left(\left(A^{-1} B\right)^{ \pm 1}, B^{ \pm 1}\right)$ or $\left(B^{ \pm 1},\left(A^{-1} B\right)^{ \pm 1}\right)$. The algorithm 
requires that at each step the generators are properly normalized. One can see that this translates to

Lemma 2.7. The algorithm replaces the normalized generators $(A, B)$ by the normalized next pair which is either $\left(\left(A^{-1} B\right)^{-1}, B\right)$ or $\left(B^{-1}, A^{-1} B\right)$.

The first type of replacement is called a linear or non-Fibonacci step, the second, a Fibonacci step (see [3] or [18]). Under Fibonacci steps, the lengths of subsequent generators as words in the original generators will grow exponentially and under non-Fibonacci steps the lengths grow linearly.

Thus we can associate to a given input to the algorithm a sequence of integers $n_{i}$, called the $F$ - sequence, where $n_{1}, n_{2}, \ldots, n_{k}$ denotes the number of consecutive non-Fibonacci or linear steps. There is a Fibonacci step between each of the consecutive $n_{i}$ linear steps.

We let $\left(\tilde{A}_{n_{i}}, \tilde{B}_{n_{i}}\right)$ denote the normalized words in $A$ and $B$ that are the output of the algorithm after the sequence of steps $n_{1}, 1, n_{2}, \ldots, 1, n_{i}, 1$ has been executed. As a corollary to lemma 2.7 we get the recursive Fibonaccilike formulas:

\section{Corollary 2.8.}

$$
\left(\tilde{A}_{n_{i+1}}, \tilde{B}_{n_{i}+1}\right)=\left(\left(\tilde{B}_{n_{i}}\right)^{-1},\left(\tilde{A}_{n_{i}}\right)^{-1}\left(\tilde{B}_{n_{i}}\right)^{n_{i+1}}\right) .
$$

That is,

$$
\tilde{A}_{n_{i+1}}=\left(\tilde{B}_{n_{i}}\right)^{-1}, \quad \tilde{B}_{n_{i+1}}=\tilde{B}_{n_{i-1}}\left(\tilde{B}_{n_{i}}\right)^{n_{i+1}}
$$

From the normalization we get that $n_{1} \neq 0$. For $j>1$ we may have $n_{j}=0$. The total number of generating pairs that the algorithm considers is $k-1+\sum_{i=1}^{k} n_{i}$.

We observe that if $n_{2} \neq 0$ and $n_{3} \neq 0$, the output of the algorithm is:

$$
\begin{aligned}
&(A, B) \rightarrow\left(\left(A^{-1} B\right)^{-1}, B\right) \rightarrow\left(\left(A^{-1} B^{2}\right)^{-1}, B\right) \rightarrow \ldots \rightarrow\left(\left(A^{-1} B^{n_{1}-1}\right)^{-1}, B\right) \\
& \rightarrow\left(B^{-1}, A^{-1} B^{n_{1}}\right) \rightarrow \\
&\left(\left(B \cdot A^{-1} B^{n_{1}}\right)^{-1}, A^{-1} B^{n_{1}}\right) \rightarrow\left(\left(B \cdot\left(A^{-1} B^{n_{1}}\right)^{2}\right)^{-1}, A^{-1} B^{n_{1}}\right) \rightarrow \ldots
\end{aligned}
$$




$$
\rightarrow\left(\left(B \cdot\left(A^{-1} B^{n_{1}}\right)^{n_{2}-1}\right)^{-1}, A^{-1} B^{n_{1}}\right) \rightarrow
$$

and

$$
\begin{gathered}
\left.\left(\left(A^{-1} B^{n_{1}}\right)\right)^{-1}, B \cdot\left(A^{-1} B^{n_{1}}\right)^{n_{2}}\right) \\
\left.\rightarrow \ldots \rightarrow\left(\left(A^{-1} B^{n_{1}} \cdot\left[B \cdot\left(A^{-1} B^{n_{1}}\right)^{n_{2}}\right)\right]^{n_{3}-1}\right)^{-1}, B \cdot\left(A^{-1} B^{n_{1}}\right)^{n_{2}}\right) \rightarrow \\
\left.\left(\left[B \cdot\left(A^{-1} B^{n_{1}}\right)^{n_{2}}\right)\right]^{-1},\left(A^{-1} B^{n_{1}} \cdot\left[B \cdot\left(A^{-1} B^{n_{1}}\right)^{n_{2}}\right)\right]^{n_{3}}\right) \ldots
\end{gathered}
$$

We can expand $\tilde{B}_{n_{i}}$ in the form

$$
B^{v_{0}} A^{-1} B^{v_{1}} A^{-1} B^{v_{2}} A^{-1} B^{v_{3}} \cdots A^{-1} B^{v_{t}}
$$

where $v_{0}=v_{0}(i)$ is 0 or 1 as $i$ is odd or even, and $v_{j}=v_{j}(i)>0$ for $j=$ $1 \ldots t=t(i)$. The $v_{j}(i)$ are called the primitive exponents for the algorithmic words.

Proposition 2.9. The primitive exponent sequence for $\tilde{B}_{n_{k}}$ can be obtained from the F-sequence $\left(n_{1}, \ldots, n_{k}\right)$ recursively as follows:

- If $k=1, \tilde{B}_{n_{1}}=A^{-1} B^{n_{1}}$, so we have $t(1)=1$ and $v_{0}(1)=0, v_{1}(1)=n_{1}$.

- If $k=2, \tilde{B}_{n_{2}}=B\left(A^{-1} B^{n_{1}}\right)^{n_{2}}$. Thus, $t(2)=n_{2}, v_{0}(2)=1$, and $v_{1}(2) \ldots v_{n_{2}}(2)=n_{1}$.

- for $k=3, t(3)=n_{3} t(2), v_{0}(3)=0$, and $v_{j}(3)=n_{1}+1, j \equiv 1 \bmod t(2)$, $v_{j}(3)=n_{1}$ otherwise.

- In general, for $k>3, t(k)=t(k-2)+n_{k} t(k-1), v_{j}(k)=v_{j}(k-2), j=$ $0, \ldots t(k-2)-1$, and for $j=t(k-2) \ldots t(k), v_{j}(k)=v_{0}(k-1)+$ $v_{1}(k-1), j=t(k-2) \equiv 0 \bmod t(k-1), v_{j}(k)=n_{1}$ otherwise.

Proof. For $k=1$, there have been $n_{1}$ linear steps and the first non-linear step. We easily read off $t(1)$ and $v_{0}(1), v_{1}(1)$. For $k=2$ we note that the first factor ends with $B^{v_{0}}=B$ while the remaining $n_{2}$ factors begin with $B^{n_{1}}$ and end with $B^{v_{0}}$.

At step $k$, we have $1+n_{k}$ factors. The exponents in the first factor repeat those of the $k-2$-nd step. The new $n_{k}$ factors combine as they do for $k=2$. Note though, that if $k$ is even, $B^{v_{0}}=i d$. 


\section{Intersections \& Reflections}

In the previous section, we related the sequence of generating pairs determined by the algorithm to special algebraic word forms. In this section we turn to the underlying geometry. Recall from the introduction, that if the axes of the given generators $(A, B)$ are disjoint, and if the group $G=<A, B>$ is discrete, then the quotient $S=U / G$ is topologically a three times punctured sphere. The axes of these generators project to geodesics $\pi\left(A x_{A}\right)$ and $\pi\left(A x_{B}\right)$ on $S$. If these geodesics are simple the algorithm stops; otherwise it continues. Therefore, if the algorithm takes any steps, $\pi\left(A x_{A}\right)$ and/or $\pi\left(A x_{B}\right)$ have self-intersections.

In the introduction, we defined the reflection lines $\left(L, L_{A}, L_{B}\right)$. They project to simple open disjoint geodesics $\pi(L), \pi\left(L_{A}\right), \pi\left(L_{B}\right)$ joining pairs of boundary disks (topological punctures) of $S$. We will see that many of the self-intersections of $\pi\left(A x_{A}\right)$ and $\pi\left(A x_{B}\right)$ lie on these open geodesics. These will be called essential intersections. We will show how the F-sequence of the algorithm describes the essential self-intersections.

As always, we let $(A, B)$ be a normalized pair of hyperbolics with disjoint axes. We continue with the notation of section 2 where $R_{M}$ denotes the reflection in the line $M$. We write compositions as products and apply the factors from right to left. We observe that $R_{R_{M}(N)}=R_{M} R_{N} R_{M}$ for any hyperbolic lines $M$ and $N$ so that

$$
\begin{gathered}
A=R_{L} R_{L_{A}}, \quad B=R_{L} R_{L_{B}}, \\
A^{-1} B=R_{L_{A}} R_{L_{B}} \text { and } A B=R_{L} R_{L_{A}} R_{L} R_{L_{B}}=R_{R_{L}\left(L_{A}\right)} R_{L_{B}} .
\end{gathered}
$$

Let $L_{B^{0}}=L_{B}$. Choose $L_{B^{2}}$ and $L_{B^{n}}$ for $n \geq 2$ so that $B^{2}=R_{L_{B^{2}}} R_{L}$ and $B^{n}=R_{L_{B^{n}}} R_{L}$.

We interpret negative powers so that

$$
B^{-1}=R_{L_{B^{-1}}} R_{L} \text { and } B^{-n}=R_{L_{B^{-n}}} R_{L} .
$$

We note that

$$
B=R_{L} R_{L_{B}}=R_{L_{B}} R_{L_{B^{2}}}=R_{L_{B^{n-1}}} R_{L_{B^{n}}} .
$$

For a normalized pair of generators $(A, B)$ we have the configuration shown in figures 4 and 5 . 
Lemma 3.1. For each $j$ with $L_{B^{j}} \cap A x_{A} \neq \emptyset$, let $p_{j}=L_{B^{j}} \cap A x_{A}$. There is a smallest integer $n$ such that $B^{-j}\left(A x_{A}\right) \cap A x_{A}=p_{j}$ for $j=1, \ldots, n$ and $B^{-j}\left(A x_{A}\right) \cap A x_{A}=\emptyset$ for $j>n$.

Proof. (See figures 4 and 5.) Let $p_{-j}=R_{L}\left(p_{j}\right)$. Then $B^{j}\left(p_{j}\right)=\left(R_{L} \circ\right.$ $\left.R_{L_{B^{j}}}\right)\left(p_{j}\right)=R_{L}\left(p_{j}\right)=p_{-j}$. As $j \rightarrow \infty, B^{-j}\left(A x_{A}\right)$ tends to the repelling fixed point of $B$. The proof follows since $A x_{A}$ and $A x_{B}$ are assumed disjoint.

Definition 3.2. If $n=n(A, B)$ is the smallest integer such that $L_{B^{n}} \cap A x_{A}=$ $\emptyset$, we call $n$ the simplicity and say $A$ is $n$ simple with respect to $B$. We say that $A$ is simple with respect to $B$ if $n(A, B)=0$ (see [15]). We define $n=n(B, A)$ analogously.

Corollary 3.3. $A$ is $B$-simple and $B$ is $A$-simple if and only if $G$ is either discrete and free or $A^{-1} B$ is elliptic.

Proof. We first observe that $A$ is $B$-simple if and only if the line $L_{B}$ does not intersect $A x_{A}$. That is, if $x \in B\left(A x_{A}\right) \cap A x_{A}$, then $R_{L_{B}(x)} \in A x_{A}$. Since $x$ and $R_{L_{B}(x)}$ lie on opposite sides of $L_{B}$, either $L_{B}$ and $A x_{A}$ coincide or intersect in a single point $y=L_{B} \cap A x_{A}$. If they coincide the group is elementary so $y$ is the only point on $B\left(A x_{A}\right) \cap A x_{A}$

Now $L, L_{A}$ and $L_{B}$ either bound, separate or $L_{A}$ and $L_{B}$ intersect in which case $A^{-1} B=R_{L_{A}} R_{L_{B}}$ is elliptic. Since $\operatorname{tr} A \geq \operatorname{tr} B, n(B, A) \neq 0$ implies $n(A, B) \neq 0$. $L_{A}, L_{B}$ and $L$ separate if and only if $n(A, B) \neq 0$.

For a stopping pair of generators we have the configuration shown in figure 2. Let $q_{A B}$ be the intersection of $A x_{A B}$ with $L$.

Lemma 3.4. (See figure 2.) When $G$ is discrete and $L_{A}, L_{B}$ and $L$ bound a region, $\pi\left(A x_{A B}\right)$ intersects itself only at the point $\pi\left(q_{A B}\right)$.

Proof. Note $A x_{B A}=A x_{(B A)^{-1}}$ and is the common perpendicular to $L_{A^{-1}}$ and $L_{B}$. Then $A x_{A^{-1} B^{-1}}=A x_{B A}$ intersects $L$ at $q_{A B}$ also. That is, $R_{L}\left(A x_{(B A)^{-1}}\right)=$ $A x_{A B}$. But $R_{L}\left(q_{A B}\right)=q_{A B}$. Since the translation length of $A B$ is twice the distance between $L_{A}$ and $L_{B^{-1}}, \pi\left(q_{A B}\right)$ is the only self-intersection point on the surface.

More generally, 


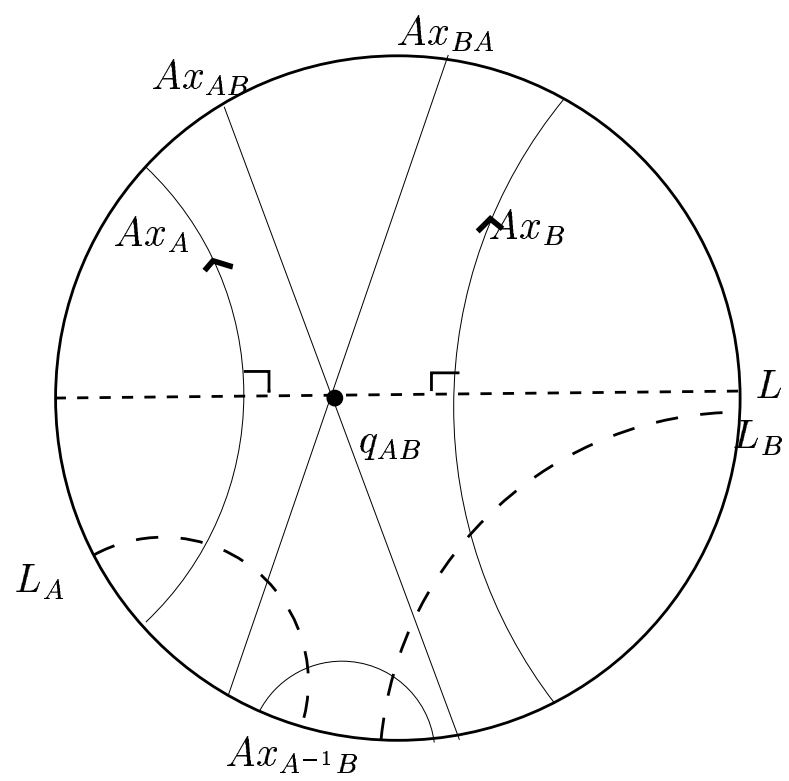

Figure 2: Reflection lines bound. Axes of $A B$ and $B A$ intersect at $q_{A B}$

Corollary 3.5. Assume $G$ is discrete and that $(A, B)$ are a pair of normalized generators for $G$ whose reflection axes bound. If $q_{i}$ denotes the point where $A x_{A^{n} B}$ intersects $L_{A^{-i}}$ for $1 \leq i \leq n$, then $\pi\left(q_{i}\right)$ is a self-intersection point of $\pi\left(A x_{A^{n} B}\right)$ lying on $\pi(L)$ if $i$ is even and on $\pi\left(L_{A}\right)$ if $i$ is odd. Further, these are the only self-intersection points on the images of the three reflection lines.

A similar statement can be made for self intersections of $A B^{n}$.

Proof. (See figure 3.) This follows from the use of perpendicularity properties together with the fact that the images of the reflection lines tessellate the plane. The axis of $A^{n} B$ is the common perpendicular to $L_{A^{-n}}$ and $L_{B}$ since $A^{n} B$ is the product of reflections in these lines. This common perpendicular must cross $L_{A^{-i}}$ for all $i$ with $0 \leq i \leq n$. For each such $i, \pi\left(L_{A^{-i}}\right)=$ $\pi(L)$ or $\pi\left(L_{A}\right)$ depending upon whether $j$ is even or odd.

Note that for every pair of generators the the three orthogonals to the axes of generators always project to same three open geodesics. Thus we make the following definition: 


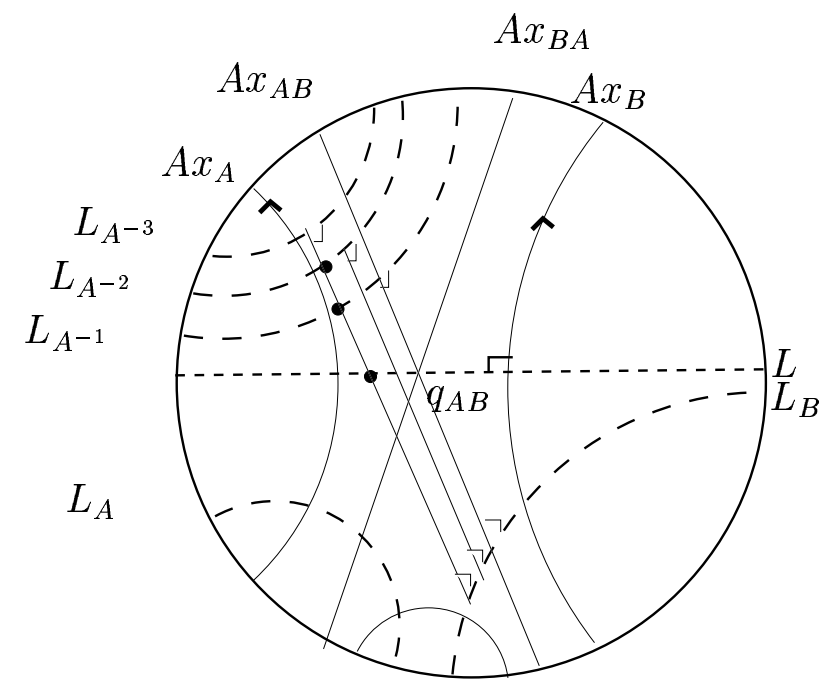

Figure 3: Reflection lines and axes of $A^{n} B$ for $n=1,2,3$

Definition 3.6. For elements $W \in G$, call self-intersections of $\pi\left(A x_{W}\right)$ that occur on images of the reflection axes, essential self-intersections.

\section{$4 \quad$ Winding and unwinding}

We want to describe how the algorithm takes us from generators with many essential intersections to simple curves by unwinding the curves. We make this idea precise.

Let $q=L \cap A x_{A}$ and $q_{A}=L_{A} \cap A x_{A}$. Then $\left[q, q_{A}\right]$ denotes the segment of $A x_{A}$ between $q$ and $q_{A}$ and, as in lemma 3.1, for any integer $m$, let $p_{m}$ denote the point on $A x_{A}$ where $L_{B^{m}}$ intersects $A x_{A}$. (See figures 4 and 5.)

Definition 4.1. We define $w(A, B)$, the winding of $A$ with respect to $B$ to be the largest integer $w \leq n(A, B)$ such that $p_{w} \in\left[q, q_{A}\right]$. We say that $A$ is $w(A, B)$-wound with respect to $B$. If $w(A, B)=0$ we say $A$ is unwound with respect to $B$. We define $w(B, A)$ analogously.

Lemma 4.2. Assume that $A$ and $B$ are normalized and that $w(A, B)=w$. Then $w\left(A^{-1} B, B\right)=w-1$ and for $0 \leq k \leq w, w\left(A^{-1} B^{k}, B\right)=w-k$. 


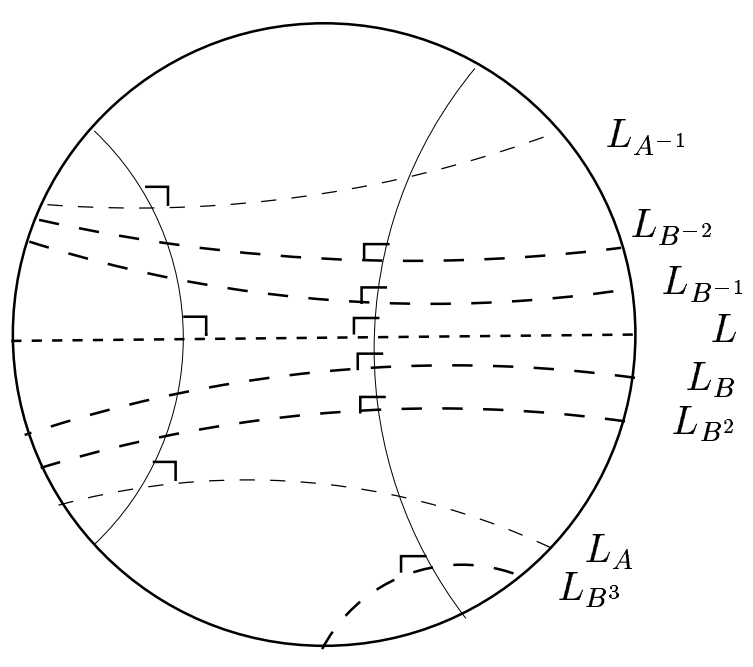

Figure 4: Reflection lines with $n_{1}=2$

Proof. (See figure 4.) We observe that $A^{-1} B^{k}$ is the product of the reflections in $L_{A}$ and $L_{B^{k}}, B$ is the product of reflections in $L_{B^{k}}$ and $L_{B^{k+1}}$ and if $k<j \leq w$, then $L_{B^{j}}$ must cross the axis of $A^{-1} B^{k}$ since $p_{j}$ will be between $p_{k}$ and $p_{w}$.

Corollary 4.3. $w(A, B)$ is the number of consecutive linear steps that the algorithm encounters beginning at $(A, B)$; the next step is a Fibonacci step.

At each Fibonacci step of the algorithm, a new unwinding process begins.

Definition 4.4. Let $A_{k}, B_{k}$ be the $k$-th ordered pair in the algorithm sequence. We let $w_{k}=w\left(A_{k}, B_{k}\right)$ be the winding of the $k$-th pair in the sequence. If the group $G=<A, B>$ is discrete and the algorithm stops at the nth generating pair, we call the sequence $\left(w_{1}, \ldots, w_{n}\right)$ the unwinding sequence of the algorithm.

Corollary 4.5. The unwinding sequence $\left(w_{1}, \ldots, w_{n}\right)$ is the same as the $F$ sequence $\left(n_{1}, \ldots, n_{k}\right)$ of consecutive linear generator steps in the algorithm; that $i s, n=k$ and $w_{i}=n_{i}$ for all $i=1, \ldots, k$.

If the algorithm begins with $(A, B)$ and ends at the pair $(C, D)$ we can consider the process of going from $(C, D)$ to $(A, B)$ as winding and the reverse process as unwinding. It is easy to see that 


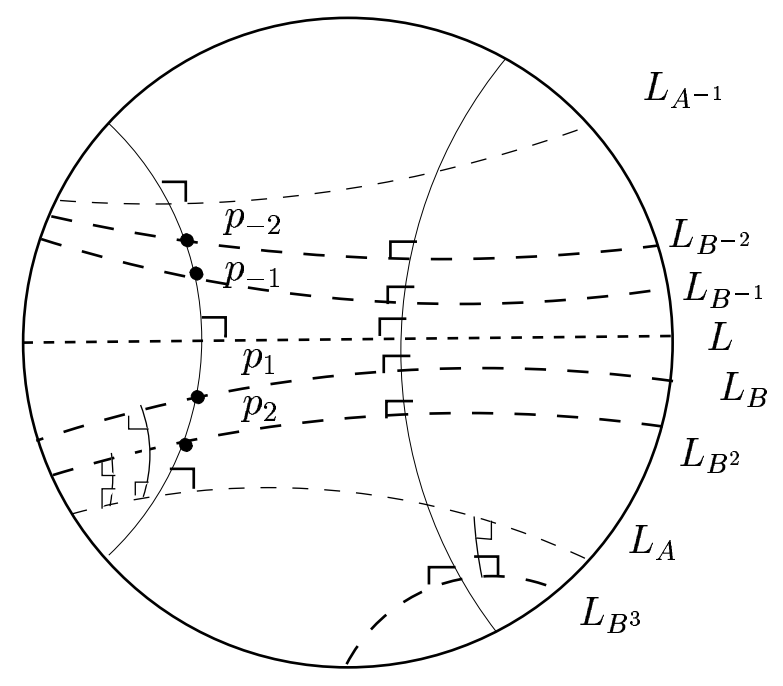

Figure 5: Reflection lines and arcs of axes of $A B^{-n}, n=1,2,3$

Proposition 4.6. If the unwinding sequence of $(A, B)$ is $\left(n_{1}, \ldots, n_{k}\right)$ then the winding sequence $(A, B)$ with respect to $(C, D)$ is $\left(n_{k}, \ldots, n_{1}\right)$.

When we complete the unwinding, the axes of the resulting elements project to simple closed geodesics on the surface as we saw in the introduction. Moreover, it is easy to see that these are these are the shortest on the surface and are unique (up to order).

Definition 4.7. The generators resulting from the algorithm are called simple generators.

\section{The Connection}

In this section, we give the relationship between the algorithm words with their unwinding sequences and the Farey words with their continued fraction expansions.

The theorem is

Theorem 5.1. Suppose $C, D$ is the ordered pair of generators obtained at some stage of the algorithm applied to the pair $A, B$ so that the unwinding 
sequence at this stage is $\left(n_{1}, \ldots, n_{k}\right)$. Let $p / q$ be the rational number with continued fraction expansion $\left[0, n_{1}, \ldots n_{k}\right]$ and $r / s$ that with $\left[0, n_{1}, \ldots, n_{k-1}\right]$. Then $(C, D)=\left(W_{r / s}, W_{p / q}\right)$ where $W_{u / v}$ denotes the $u / v$ Farey word on $A$ and $B$.

Equivalently,

Theorem 5.2. Suppose $C=W_{p / q}(A, B), D=W_{r / s}(A, B)$ are the new generators obtained at some stage of the algorithm applied to $(A, B)$ and suppose the corresponding continued fractions are, $p / q=\left[n_{0}, n_{1}, \ldots, n_{k}\right]$, $r / s=\left[n_{0}, n_{1}, \ldots, n_{k-1}\right]$. Denote the approximating fractions by $p_{m} / q_{m}=\left[n_{0}, n_{1}, \ldots, n_{m}\right]$. Then we have the recursive formulas:

$$
p_{m+1}=n_{m+1} p_{m}+p_{m-1} ; q_{m+1}=n_{m+1} q_{m}+q_{m-1}
$$

with

$$
p_{0}=n_{0}, q_{0}=1, p_{1}=n_{0} n_{1}+1, q_{1}=n_{1} .
$$

The words in the algorithm (up to inverses) are given by

$$
W_{1 / 0}=A^{-1}, W_{0 / 1}=B
$$

and

$$
W_{p_{m+1} / q_{m+1}}=\left(W_{p_{m} / q_{m}}\right)^{n_{m+1}} W_{p_{m-1} / q_{m-1}}
$$

or $n$

$$
W_{p_{m+1} / q_{m+1}}=W_{p_{m-1} / q_{m-1}}\left(W_{p_{m} / q_{m}}\right)^{n_{m+1}}
$$

as $m$ is odd or even.

Proof. The recursive formulas for $p_{m}, q_{m}$ can be found in [5].

Because of our normalization that $\operatorname{tr} A>\operatorname{tr} B$, the first group of linear steps replaces $A$ by $A^{-1} B^{j}$ for $j=1 \ldots, n_{1}-1$. In the way we have set up the Farey words, these correspond to rationals in the interval [0,1]. Any Farey neighbor of a point in $[0,1]$ is clearly again in $[0,1]$. Thus, in the continued fraction expansion of rationals corresponding to algorithm words at the first step and beyond, we always have $n_{0}=0$.

The proof follows from the construction of Farey words in theorem 2.3 where the right factor corresponds to the smaller neighbor and the fact that the approximating rationals $p_{m} / q_{m}$ approach $p / q$ alternately from left and right. Therefore within the same group of linear steps, the multiplication is always from the same side; it changes with the Fibonacci step which corresponds to the next approximating factor. 
One can run the algorithm forwards and backwards. We have the following connection:

Theorem 5.3. Suppose $A, B$ are the original generators and $C=W_{p / q}(A, B)$, $D=W_{r / s}(A, B)$ are the simple generators, obtained from the algorithm. If $p / q=\left[n_{1}, \ldots, n_{k}\right], r / s=\left[n_{1}, \ldots n_{k-1}\right]$ are the continued fraction expansions respectively, then applying the recursive formulas above with $\left[-n_{k}, \ldots,-n_{1}\right]$, we obtain, up to choice of labelling, $A=W_{-p / r}\left(D^{-1}, C\right)$ and $B=W_{-q / s}\left(D^{-1}, C\right)$.

Proof. In the Farey diagram the steps correspond to products of the matrices $S=\left(\begin{array}{cc}1 & -1 \\ 0 & 1\end{array}\right)$ and $T=\left(\begin{array}{ll}0 & 1 \\ 1 & 0\end{array}\right)$ so that $W_{p / q}=S^{n_{0}} T S^{n_{1}} T \ldots S^{n_{r}}=\left(\begin{array}{ll}p & r \\ q & s\end{array}\right)$. This matrix maps the hyperbolic line $(0, \infty)$ to the line $(p / q, r / s)$. Reversing the algorithm means taking the inverse matrix and thus reversing the order (and signs) of the $n_{i}$.

We remind the reader that the inverse matrix to $\left(\begin{array}{ll}p & r \\ q & s\end{array}\right)$ is $\left(\begin{array}{cc}s & -r \\ -q & p\end{array}\right)$ The continued fractions of $p / q$ and $-r / p$ are essentially reverses of each other (up to the usual ambiguity and the first digits).

\section{Formulas for essential self-intersection}

In this section we find a formula for the number of essential self-intersections of a primitive element of a discrete hyperbolic group $G$ whose quotient $U / G$ is a three holed sphere $S$. We begin with a generating pair $(A, B)$ for $G$ and we assume both generators are simple. Since we are going in the winding direction here, that is, the reverse direction of the algorithm, we start with $W_{1 / 0}=A, W_{0 / 1}=B$ and $W_{-1 / 1}=A^{-1} B$. We let $W=W_{p / q}(A, B)$ be the given primitive word and denote the number of essential self-intersections of $W$ by $I(W)$ or $I_{p / q}$. As we saw above, the continued fraction of $p / q$ gives us the winding sequence for $W$.

We begin by noting

$$
I(A)=0, I(B)=0, I\left(A^{-1} B\right)=0 .
$$

After 1-linear step we have normalized generators $(B, A B)$ and

$$
I(A B)=1, I(B)=0, I\left(A B^{2}\right)=2 .
$$


These are easy to check by hand.

After $n_{1}$ linear steps we have $I(B)=0, I\left(A B^{n_{1}}\right)=n_{1}$. For the first Fibonacci step we have normalized generators $\left(\left(A B^{n_{1}-1}\right)^{-1},\left(A B^{n_{1}}\right)^{-1}\right.$. Now we get

$$
I\left(\left(A B^{n_{1}}\right)^{-1}\left(A B^{n_{1}-1}\right)^{-1}=I\left(A B^{n_{1}-1} A B^{n_{1}}\right)=1+n_{1}+n_{1}-1\right.
$$

The general rule is

Theorem 6.1. Let $p / q=\left[n_{0}, \ldots n_{k}\right]$. Then $I_{p_{m+1} / q_{m+1}}=1+n_{m+1} I_{p_{m} / q_{m}}+$ $I_{p_{m-1} / q_{m-1}}$

To prove this theorem we look both on $S$ and in the covering $U$. To simplify notation we write $\gamma_{p / q}$ for $\pi\left(A x_{W_{p / q}}\right)$ so that $\gamma_{\infty}=\pi\left(A x_{A}\right)$ and $\gamma_{0}=\pi\left(A x_{B}\right)$. These are simple geodesics going around two of the holes. The simple geodesic around the third hole is $\gamma_{-1}$. We also set $l_{a b}=\pi(L), l_{a}=$ $\pi\left(L_{A}\right)$ and $l_{b}=\pi\left(L_{B}\right)$. Note that $l_{a b}$ is orthogonal to $\gamma_{\infty}$ and $\gamma_{0}$ and disjoint from $\gamma_{-1}$ and similarly for $l_{a}$ and $l_{b}$. See figure 7 .

Lemma 6.2. The projection $\gamma_{p / q}$ goes $p$ times around $\gamma_{\infty}$ and $q$ times around $\gamma_{0}$

Proof. The proof is trivial since $W_{p / q}$ contains $p A^{\prime}$ 's and $q$ B's

Lemma 6.3. If $p$ is odd then $\gamma_{p / q}$ is the loop farthest out (in the hyperbolic metric) along the hole bounded by $\gamma_{\infty}$ and it intersects $l_{a}$ orthogonally whereas if $p$ is even, the farthest out loop intersects $l_{a b}$ orthogonally. Similarly for $q$ and $l_{b}$

Proof. (See figure 7) The reflections of $U$ through the reflection lines descend to symmetries of $S$ through their projections. These symmetries map $\gamma_{p / q}$ to itself and the angle $\gamma_{p / q}$ makes at an intersection with one of the $l$-lines is sent to its negative. Thus if this angle is not $\pi / 2$, the reflected seqments of $\gamma_{p / q}$ at this point cross each other but if it is $\pi / 2$ there is only one such segment and no self-intersection. Since there are finitely many loops around $\gamma_{\infty}$ there is one farthest out and it intersects itself on only one $l$ line; its other (furthest out) intersection with an $l$ line is orthogonal.

If $p=1, q=1$, the lemma follows from lemma 3.4 by projection. Using corollary 3.5, the other cases follow by induction.

Lemma 6.4. If $\gamma_{p / q}$ intersects $l_{a b}$ orthogonally on the $\gamma_{0}$ side, it intersects $l_{a}$ orthogonally on the $\gamma_{\infty}$ side and vice versa. 
Proof. Since $(p, q)=1$, at least one of $p, q$ is odd. Now apply the previous lemma.

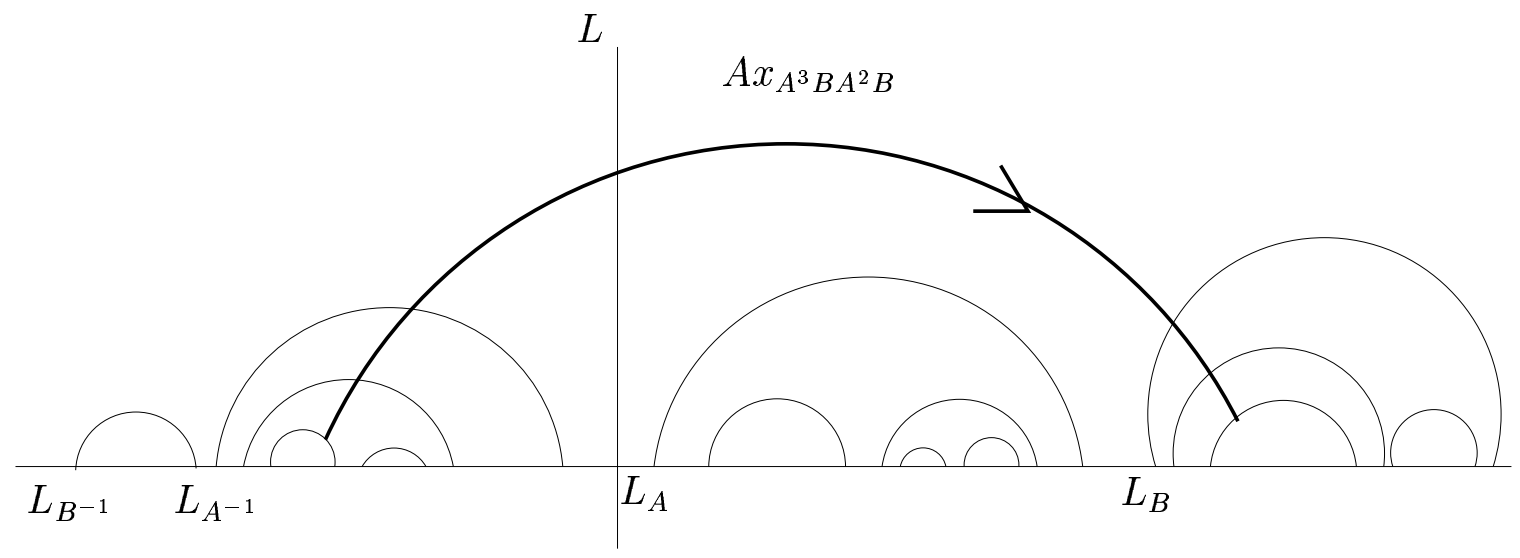

Figure 6: Finding essential intersections. The reflections lines are light solid here. The axis of $A^{3} B A^{2} B$ is dark solid

Proof. Proof of the theorem. (See figure 6) The proof proceeds by induction on the Farey level of $p / q$. The first step is obviously true. Then assume it is true for level $m$ and take the next product.

Here is the induction step. See figure 6. Consider $\gamma_{p_{m} / q_{m}}$ and $\gamma_{p_{m-1} / q_{m-1}}$. By the above lemmas, there is exactly one $l$ line such that both are orthogonal to it. Assume for argument's sake that it is $l_{a b}$. Consider lifts to the upper half plane model of $U$ as follows. Let $L$, the lift of $l_{a b}$, be the imaginary axis with $L_{A}, L_{B}$ to the right and $L_{\bar{A}}, L_{\bar{B}}$ to the left. Draw a fundamental segment $S_{m}$ for $W_{p_{m} / q_{m}}$ on the left and another $S_{m-1}$ for $W_{p_{m-1} / q_{m-1}}$ on the right. Each segment ends on an image $L_{m}$ or $L_{m-1}$ of $L ; S_{m}$ intersects $I_{m}$ images of $L, L_{A}, L_{B}$ and $S_{m-1}$ intersects $I_{m-1}$ images of $L, L_{A}, L_{B}$. Then the axes of $W_{p_{m+1} / q_{m+1}}=W_{p_{m} / q_{m}} W_{p_{m-1} / q_{m-1}}$ and $W_{p_{m-1} / q_{m-1}} W_{p_{m} / q_{m}}$ intersect $L$ between its intersections with the fundamental segments. A fundamental segment for $W_{p_{m+1} / q_{m+1}}$ goes from $L_{m}$ to $L_{m-1}$. Thus it intersects $1+I_{p_{m} / q_{m}}+I_{p_{m-1} / q_{m-1}}$ images of the $L$ lines as claimed. 


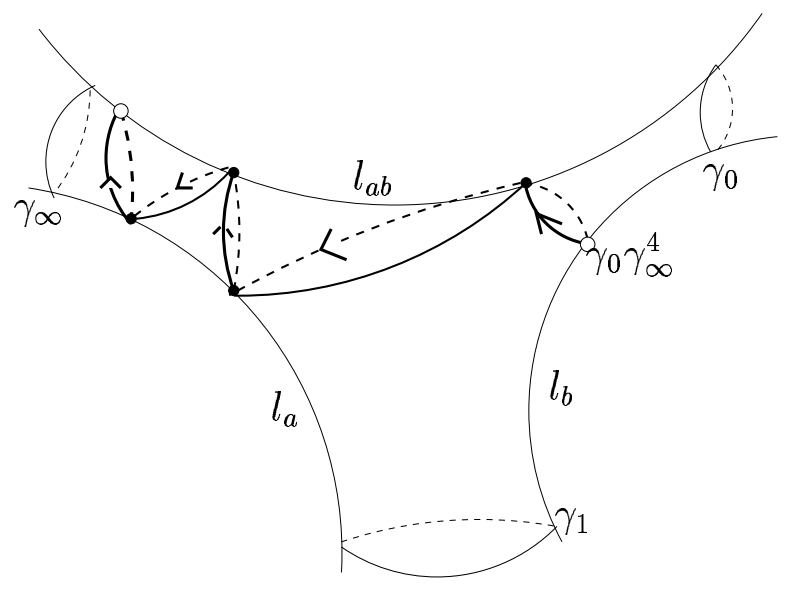

Figure 7: The winding of $\gamma_{0} \gamma_{\infty}^{4}$. The 4 essential intersections are marked with a large dot

Remark 6.5. The $p$ loops of $\gamma_{p / q}$ about $\gamma_{\infty}$ and $q$ loops about $\gamma_{0}$ are wrapped according to the continued fraction expansion: one loop around $\gamma_{\infty}$ is followed by $n_{1}$ loops around $\gamma_{0}$; then, changing direction, this is followed by $n_{2}$ non-simple loops around $\gamma_{\infty} \gamma_{0}^{n_{1}}$ and, changing direction again, $n_{3}$ non-simple loops around $\left(\gamma_{\infty} \gamma_{0}^{n_{1}}\right)^{n_{2}}$ etc.

The computational complexity of the discreteness algorithm must take into account a number of items, including the maximal translation length of the generators, the number of generating pairs and the lengths of the words in the original generators ([3] and [18]). The latter two can be computed from the algorithm sequence, that is, the unwinding sequence $\left[n_{1}, \ldots, n_{k}\right]$. It is in this sense that it is appropriate to term the complexity of a curve its unwinding sequence. This leads us to define

Definition 6.6. If $\gamma_{p / q}$ is the projection of the axis of a word $W_{p / q}$ in the simple generators. Then the complexity of $\gamma_{p / q}$ is defined as $\left[n_{1}, \ldots, n_{k}\right]$ the continued fraction expansion of $p / q$, or equivalently the unwinding sequence. 


\section{Summary}

Given $G=<A, B>$ such that the algorithm determines $G$ is hyperbolic and discrete, it finds a unique normalized pair of generators $(C, D)$ for $G$ with the properties that the axes of $C, D$ and $C^{-1} D$ project to the only simple closed geodesics on $S=U / G$ and that the projections are the shortest closed geodesics on $S$.

The algorithm determines a sequence of positive integers $\left(n_{1}, \ldots, n_{k}\right)$ that can be interpreted in many ways. We sum up our results in the following theorem:

Theorem 7.1 (Summary of results). 1. If the F-sequence of the algorithm is $\left(n_{1}, \ldots, n_{k}\right)$, let $p / q$ be the rational number with continued fraction expansion $\left[n_{1}, \ldots, n_{k}\right]$ and $r / s$ the rational number with continued fraction expansion $\left[n_{1}, \ldots, n_{k-1}\right]$. Let $(C, D)$ be the stopping pair for the algorithm. Then $C$ is the Farey word $W_{r / s}$ and $D$ is the Farey word $W_{p / q}$.

2. The numbers of consecutive linear steps of the algorithm determine its F-sequence $\left(n_{1}, \ldots, n_{k}\right)$.

3. There is a Fibonacci step between each pair $\left(n_{i}, n_{i+1}\right)$ of consecutive sets of linear steps. There are $k-1$ Fibonacci steps. The Farey level of $p / q$ is $\sum_{1}^{k} n_{i}$ and is equal to the total number of linear steps the algorithm executes.

4. The sequence $\left(n_{1}, \ldots, n_{k}\right)$ is also the unwinding sequence of the algorithm.

5. Reversing the steps of the algorithm determines the winding sequence $\left(\tilde{w}_{1}, \ldots, \tilde{w}_{k}\right)$ of $(A, B)$ with respect to $(C, D)$. We have $\tilde{w}_{j}=-n_{k-j}$ for all integers $j$.

6. When $G$ is discrete the algorithm finds the three shortest geodesics on the surface which are all simple.

7. The essential self-intersections of the projections of the axes of the original generators can be computed from the inductive formula $I_{p_{m+1} / q_{m+1}}=$ $1+I_{p_{m} / q_{m}}+I_{p_{m-1} / q_{m-1}}$ where $p_{m} / q_{m}$ is defined by the continued fraction $\left[n_{1}, \ldots, n_{m}\right]$. 
8. Associate primitive pairs of generators are in one to one correspondence with pairs $p / q, r / s$ of Farey neighbors of rational numbers. Their primitive exponents can be found inductively from the continued fraction expansion. If $C=W_{r / s}(A, B), D=W_{p / q}(A, B)$ then $p / q$ has the continued fraction expansion $\left[n_{0}, n_{1}, \ldots, n_{k}\right]$ with $n_{0}=0$ and the remaining continued fraction entries are equal to the corresponding remaining F-sequence entries.

9. $(C, D)$ are an associate primitive pair of generators and each can be written in its primitive form:

$$
A^{u_{1}} B^{v_{1}} A^{u_{2}} \ldots B^{v_{t}}
$$

for some $t$, where the $u_{i}$ are all equal to each other and are all either 1 or -1 , the $v_{i}$ all have the opposite sign to the $u_{i}$, and $\left|v_{i}-v_{i+1}\right| \leq 1$. The $v_{i}$ 's are the primitive exponents. They determine an F-sequence $\left(n_{1}, \ldots, n_{k}\right)$ and a corresponding rational $p / q$.

10. The numbers of consecutive linear steps of the algorithm determine its $F$-sequence $\left(n_{1}, \ldots, n_{k}\right)$ and these in turn determine the primitive exponents $v_{i}$ of the primitive form.

\section{Groups with parabolics}

The reflection axes, $L, L_{A}, L_{B}$ can be defined if one or both of the generators are parabolic. For $A$ hyperbolic and $B$ parabolic define $L$ to be the perpendicular from the boundary fixed point of $B$ to $A x_{A}$; if $A$ and $B$ are both

parabolic, $L$ is the line connecting their fixed points. With this definition of $L$ and a normalization for $A$ and $B$, the other two reflection axes are well defined. In all cases, the group $G$ is discrete and free if the reflection axes bound a region. The group is either not discrete or not free if two of the reflection axes intersect interior to $U$.

More generally, if the group $G$ contained a primitive parabolic generator, then at step $n$ for some $n$ the algorithm might encounter a pair of hyperbolics $A_{n}, B_{n}$ where the reflection lines $L_{A_{n}}, L_{B_{n}}$ meet on the boundary of $U$; this would imply $A_{n}^{-1} B_{n}$ is parabolic.

Thus at any given step one can still consider whether the reflection axes either intersect or bound a region. If $A$ is hyperbolic with $B$ parabolic, 
there is still a winding of $A$ about (the fixed point of) $B, w(A, B)$ and the simplicity of $A$ with respect to $B, n(A, B)$ is still well defined. The winding on the surface is around the puncture.

The algorithm however, encounters no Fibonacci steps. That is, after $w(A, B)$ steps, either the algorithm decides that $G$ is discrete or not discrete or not free or it replaces the pair $(A, B)$ by the pair $\left(\left(A^{-1} B^{w(A, B)}\right)^{-1}, B\right)$. Then either $\operatorname{tr} A^{-1} B^{w(A, B)} B \leq-2$, in which case the group is discrete and free, or the group is not free.

At the discrete stopping point, we have one of the following geometric situations: (i) $A$ hyperbolic, $B$ parabolic, $A^{-1} B$ hyperbolic; $S$ has a puncture corresponding to $B, \pi\left(A x_{A}\right)$ and $\pi\left(A X_{A^{-1} B}\right)$ are disjoint simple closed curves on $S$ and are the shortest curves on $S$. (ii) $A$ hyperbolic, $B$ parabolic, $A^{-1} B$ parabolic; $S$ has two punctures corresponding to $B$ and $A^{-1} B$ and $\pi\left(A x_{A}\right)$ is the unique simple closed geodesic on $S$ and the shortest closed geodesic. (iii) $A$ parabolic, $B$ parabolic, $A^{-1} B$ hyperbolic; again $S$ has two punctures, this time corresponding to $A$ and $B$ and $\pi\left(A x_{A^{-1} B}\right)$ is the unique simple geodesic on $S$ and is the shortest curve on $S$. (iv) $A$ parabolic, $B$ parabolic, $A^{-1} B$ parabolic; $S$ is a three times punctured sphere. It has no simple geodesics.

The statements in theorems 7.1, 5.1 and 5.2 remain true. All of the statements about intersections, winding, and unwinding make sense and the proofs cover the siutations that arise when one or both of the generators are parabolic. For example, the axis of $A B$ is still the common perpendicular to $R_{L}\left(L_{A}\right)$ and $L_{B}$ and the single self intersection point of $\pi\left(A x_{A B}\right)$ is still the projection of the intersection of $L$ with the axis of $A B$.

Corollary 8.1. Theorems 5.1, 5.2, 6.1 and 7.1 remain valid if one or both of the initial and/or final generators are parabolic.

Remark 8.2. The concept of winding and unwinding one curve about a point can be extended to apply to groups that contain elliptic elements or even generated by elliptic elements. The winding and unwinding numbers need to be adjusted to take into account the order of the elliptic and how it is self-wound. 


\section{References}

[1] A. F. Beardon, The geometry of discrete groups, Springer-Verlag 1983.

[2] J. Gilman, Two generator discrete subgroups of $P S L(2, R)$, Memoirs of the AMS 117 No. 561 (1995).

[3] J. Gilman, Algorithms, Complexity and Discreteness Criteria in $P S L(2, C)$, Journal D'Analyse Mathematique, Vol 73, (1997), 91-114.

[4] J. Gilman and B. Maskit, An algorithm for two-generator discrete groups, Mich. Math. J. 38 (1991) 13-32.

[5] G.H. Hardy and E. M. Wright, An introduction to the theory of numbers Oxford, Clarendon Press 1938.

[6] T. Jørgensen, On discrete groups of Möbius transformations, Amer. J. Math. 78 (1976) 739-749.

[7] A.W. Knapp, Doubly generated Fuchsian groups, Michigan Math. J. 15 (1968) 289-304.

[8] Keen, Linda, Canonical Polygons for Finitely Generated Fuchsian Groups. Acta Mathematica 115 (1966).

[9] Keen, Linda, Intrinsic Moduli on Riemann Surfaces. Annals of Mathematics 84 \#3 (1966), 404-420.

[10] (with C. Series) Pleating Coordinates for the Maskit Embedding of Teichmüller space for a punctured torus, Topology, Vol. 32 \#4, (1993),719749 .

[11] (with C. Series) On the Riley slice of Schottky space, Proc. London Math. Soc. 69 (1994) 72-90.

[12] Magnus, Karass, and Solitar, Combinatorial Group Theory, Wiley \& Sons (1966).

[13] B. Maskit, Kleinian groups, Springer-Verlag 1987.

[14] P. Matelski, The classification of discrete 2-generator subgroups of $\operatorname{PSL}(2, \mathbb{R})$, Israeli J. Math 42 (1982), 309-317. 
[15] N. Purzitsky, Real two dimensional representations of discrete free products, Math Z. 127 (1972), 95-104.

[16] Rosenberger, G., All generating pairs for all two-generator Fuchsian Groups, Arch. Math 46 (1986), 198-204.

[17] Series, Caroline The Geometry of Markoff Numbers, Math. Intelligencer 7 \#3,(1985), 20-29.

[18] Yicheng Jiang, Polynomial Complexity of the Gilman-Maskit Discreteness Algorithm Ann. Acad. Sci. Fenn. Math., 26 (2001), 375-390.

e-mail:

gilman@andromeda.rutgers.edu

linda@alpha.lehman.cuny.edu 